\title{
Meiotic silencing and fragmentation of the male germline restricted chromosome in zebra finch
}

\author{
Sam Schoenmakers • Evelyne Wassenaar • \\ Joop S. E. Laven • J. Anton Grootegoed • \\ Willy M. Baarends
}

Received: 30 September 2009 /Revised: 21 December 2009/Accepted: 11 January 2010 /Published online: 17 February 2010

(C) The Author(s) 2010. This article is published with open access at Springerlink.com

\begin{abstract}
During male meiotic prophase in mammals, $\mathrm{X}$ and $\mathrm{Y}$ are in a largely unsynapsed configuration, which is thought to trigger meiotic sex chromosome inactivation (MSCI). In avian species, females are ZW, and males ZZ. Although Z and $\mathrm{W}$ in chicken oocytes show complete, largely heterologous synapsis, they too undergo MSCI, albeit only transiently. The $\mathrm{W}$ chromosome is already inactive in early meiotic prophase, and inactive chromatin marks may spread on to the $\mathrm{Z}$ upon synapsis. Mammalian MSCI is considered as a specialised form of the general meiotic silencing mechanism, named meiotic silencing of unsynapsed chromatin (MSUC). Herein, we studied the avian form of MSUC, by analysing the behaviour of the peculiar germline restricted chromosome (GRC) that is present as a single copy in zebra finch spermatocytes. In the female germline, this chromosome is present in two copies, which normally synapse and recombine. In contrast, during male meiosis, the single GRC is always eliminated. We found that the GRC in the male germline is silenced from early leptotene onwards, similar to the $\mathrm{W}$ chromosome in avian oocytes. The GRC remains largely unsynapsed throughout meiotic prophase I, although
\end{abstract}

Communicated by P. Cohen

Electronic supplementary material The online version of this article (doi:10.1007/s00412-010-0258-9) contains supplementary material, which is available to authorized users.

S. Schoenmakers $\cdot$ J. S. E. Laven

Department of Obstetrics and Gynaecology,

Erasmus MC-University Medical Center,

PO BOX 2040, 3000 CA Rotterdam, The Netherlands

S. Schoenmakers $\cdot$ E. Wassenaar · J. A. Grootegoed

W. M. Baarends $(\triangle)$

Department of Reproduction and Development,

Erasmus MC-University Medical Center,

PO BOX 2040, 3000 CA Rotterdam, The Netherlands

e-mail: w.baarends@erasmusmc.nl patches of SYCP1 staining indicate that part of the GRC may self-synapse. In addition, the GRC is largely devoid of meiotic double strand breaks. We observed a lack of the inner centromere protein INCENP on the GRC and elimination of the GRC following metaphase I. Subsequently, the GRC forms a micronucleus in which the DNA is fragmented. We conclude that in contrast to MSUC in mammals, meiotic silencing of this single chromosome in the avian germline occurs prior to, and independent of DNA double strand breaks and chromosome pairing, hence we have named this phenomenon meiotic silencing prior to synapsis (MSPS).

\section{Introduction}

In vertebrate species, male and female meiotic prophase starts with the introduction of genome wide meiosisspecific DNA double strand breaks (DSBs) during (pre) leptotene. Meiotic DSBs are detected and repaired by the homologous recombination repair machinery, using the homologous chromosomal partner as template (reviewed in Zickler 2006 and Keeney et al. 1997). The DSB repair protein RAD51 and its meiosis-specific paralogue DMC1, rapidly form filaments on the $3^{\prime}$ end single-strand DNA tails that result from processing of the meiotic DSBs, and these filaments can be identified as distinct RAD51 foci (reviewed in Neale and Keeney 2006).

The presence of RAD51 and DMC1 mark the initiation of meiotic recombination, which involves homologous chromosome alignment, pairing, synapsis and the formation of crossovers. All these steps are necessary to obtain correct DSB repair and subsequent proper segregation of the chromosomes during the meiotic divisions, with the haploid gametes as a final result. With progression of meiotic prophase (zygotene), the number of RAD51 foci gradually 
decreases to almost zero on the synapsed autosomal chromosomes at early pachytene (Moens et al. 1997). During synapsis, the synaptonemal complex (SC) keeps the homologous chromosomes physically connected along their axes (reviewed in Zickler 2006) and ultimate complete synapsis is essential for normal continuation of the meiotic prophase. Failure of homologous chromosomes to reach full synapsis leads to meiotic arrest and apoptosis (reviewed in Turner 2007). The male mammalian $X$ and $Y$ chromosomes and the female avian $\mathrm{Z}$ and $\mathrm{W}$ sex chromosomes form a pair but are largely nonhomologous. Therefore, these sex chromosomes are confronted with a pairing problem during meiosis. The avian $\mathrm{Z}$ and $\mathrm{W}$ have solved this problem by wrapping the much longer $\mathrm{Z}$ chromosome around the short $\mathrm{W}$ chromosome during pachytene, resulting in a brief state of heterologous, but complete synapsis at mid pachytene (Solari 1992; Schoenmakers et al. 2009). Hereafter, during late pachytene, the Z and W start to desynapse again (Solari 1992; Schoenmakers et al. 2009). Although the mammalian XY pair shows extensive (heterologous) synapsis in early pachytene, this is followed by gradual desynapsis, and only the small homologous pseudoautosomal region remains synapsed in late pachytene, leaving the rest of the $\mathrm{X}$ and $\mathrm{Y}$ chromosomal arms unsynapsed (Tres 1977). In addition to the pairing problem, the nonhomologous sex chromosomes also face the presence of persistent unrepaired meiotic DSBs. These arise because the homologous template needed for recombination repair is lacking, and repair via the sisterchromatid is most likely repressed (Roeder 1997). Consequently, RAD51 foci persist on the unsynapsed region of the mammalian X chromosome until late pachytene (Ashley et al. 1995), and reappear on the desynapsing avian $Z$ chromosome at mid-late pachytene (Schoenmakers et al. 2009).

During male mammalian meiotic prophase, the unsynapsed arms of the $\mathrm{X}$ and $\mathrm{Y}$ chromosomes are detected, and meiotic sex chromosome inactivation (MSCI) causes transcriptional silencing of the sex chromosomes, resulting in the formation of the heterochromatic XY body (Monesi 1965; Turner et al. 2006). Initiation and maintenance of MSCI in mammals is always associated with the combination of persistent DSBs and asynapsis. Recently, we found that, although the avian ZW pair synapses completely, it is still subjected to a transient form of MSCI (Schoenmakers et al. 2009).

MSCI is considered as a more efficient and specialised form of the general mechanism of meiotic silencing of unsynapsed chromatin (MSUC), which transcriptionally inactivates all unsynapsed (nonhomologous autosomal) chromatin. MSCI always silences the $\mathrm{X}$ and $\mathrm{Y}$ chromosomes, but MSUC in mammals can be circumvented through heterologous (self-)synapsis, which probably conceals the nonhomologous regions (reviewed in Turner 2007). When autosomal chromosomal regions without a homologous partner remain unsynapsed, these regions become silenced by MSUC and also show persistent RAD51 foci indicative of unrepaired DSBs (Plug et al. 1998; Turner et al. 2005).

In birds, the mechanism of MSCI may be essentially different from that in mammals. Heterochromatinisation by hypermethylation of H3K9 (Schoenmakers et al. 2009) implying the inactivation of $\mathrm{W}$, precedes the introduction of DSBs and initiation of synapsis of homologous chromosomes, indicating a DNA repair and synapsis-independent recognition of the single $\mathrm{W}$ chromosome. Indeed, the $\mathrm{ZW}$ pair is silenced despite its complete heterologous synapsis. We have proposed that the heterochromatin of $\mathrm{W}$ spreads onto $\mathrm{Z}$ during the intimate wrapping of $\mathrm{Z}$ around $\mathrm{W}$ during pachytene (Schoenmakers et al. 2009). However, in chicken as well as mammalian meiotic cells, there appears to be a link between the presence of unrepaired DSBs and transcriptional silencing. In mammals, this is already apparent at the initiation of silencing, whereas in chicken oocytes, such a link is observed later, when $\mathrm{Z}$ and $\mathrm{W}$ desynapse during late pachytene. At this stage, RAD51 foci reappear on the unsynaped part of $Z$, together with accumulation of $\gamma \mathrm{H} 2 \mathrm{AX}$ and ubiquitylated H2A on these parts (Schoenmakers et al. 2009). These histone modifications may help to maintain $Z$ chromosome silencing until the DSBs have been repaired.

Since both $\mathrm{Z}$ and $\mathrm{W}$ are silenced in the female germline of birds by MSCI, we next asked how the mechanism of MSUC operates in avian species. To answer this question, we chose the zebra finch (Taeniopygia guttata) as a model system. This avian species carries a peculiar additional chromosome which is only present in the germline, named germline restricted chromosome (GRC). The GRC is present as a single copy in the male germline, whereas two copies are detected in most oocytes (Pigozzi and Solari 1998; Pigozzi and Solari 2005). In the female germline, the two copies synapse and form a bivalent, which normally participates in pairing and meiotic recombination (Pigozzi and Solari 1998; Pigozzi and Solari 2005). In the male germline, the single GRC lacks a homolog and remains unsynapsed. In addition, it is heterochromatic, resembling the mammalian XY body, and Pigozzi and Solari (1998, 2005) reported that the GRC is eliminated from the nucleus around metaphase I. To investigate the general process of meiotic silencing in birds, we analysed the behaviour of the GRC in the male and female zebrafinch germline.

\section{Results}

The GRC forms a heterochromatic body at the end of preleptotene that persists until early diplotene

Two previous reports described the formation of a $4^{\prime}, 6^{\prime}$ diamindino-2-phenylindole (DAPI) dense body (DDB) 
during meiotic prophase in zebra finch spermatogenesis (Pigozzi and Solari 1998; Pigozzi and Solari 2005). We observed that the DDB or GRC body almost always localises in the periphery of the nucleus and is, except in diplotene, easily identifiable as the intense DAPI field that clearly stands out from the rest of the chromosomes (Fig. 1a). To discriminate between the different substages of meiotic prophase, we immunostained for the presence of the lateral element of the synaptonemal complex, using antibodies targeting SYCP3. At preleptotene, SYPC3 foci start to appear in the nucleus. A DDB is visible in only part of the preleptotene nuclei (Fig. 3a), indicating that preleptotene is a transitory stage during which the DDB is formed. At leptotene and zygotene, a DDB is visible in all nuclei and small SYCP3 fragments occasionally localised in the DDB, while thick and long SC axial elements are present on assembling chromosome pairs in the rest of the nucleus. In pachytene, the GRC chromosomal axis is coated by SYCP3, but the signal is not always continuous. The axial SYCP3 element of the GRC is relatively thin compared to the SYCP3 axes associated with the other chromosomes, due to the fact that only one axial element has been assembled.

Despite the absence of a pairing partner for the GRC, we sometimes observed patches of SYCP1 assembly on the GRC (Fig. 1c), most likely representing small regions of self-synapsis.

$\gamma \mathrm{H} 2 \mathrm{AX}$ does not mark the GRC during meiotic prophase, but accumulates on the eliminated micronucleus

It is well-known that shortly after the formation of DSBs, histone $\mathrm{H} 2 \mathrm{AX}$ is phosphorylated at serine 139, forming $\gamma \mathrm{H} 2 \mathrm{AX}$ (Mahadevaiah et al. 2001; Rogakou et al. 1998). In addition, $\gamma \mathrm{H} 2 \mathrm{AX}$ marks the silenced sex chromosomes from late zygotene until diplotene in mouse (XY) and marsupial (XY) spermatocytes. In female (ZW) chicken oocytes, it marks the $\mathrm{Z}$ chromosome during desynapsis from the $\mathrm{W}$, from late pachytene until diplotene (Schoenmakers et al. 2009). In zebra finch spermatocytes, $\gamma \mathrm{H} 2 \mathrm{AX}$ was found to accumulate in leptotene nuclei, mainly confined to the areas where SYCP3 had already assembled, most likely representing sites of meiosis-specific induced DSBs. Also, in zygotene and pachytene, $\gamma \mathrm{H} 2 \mathrm{AX}$ mostly colocalises with the SYCP3 filaments. In pachytene, only a few $\gamma \mathrm{H} 2 \mathrm{AX}$ foci and some larger $\gamma \mathrm{H} 2 \mathrm{AX}$-positive areas remained. In diplotene, the $\gamma \mathrm{H} 2 \mathrm{AX}$ signal was found to increase and to disperse throughout the nucleus (Fig. 1a).

In early meiotic prophase, the area of the GRC was found to be mostly devoid of $\gamma \mathrm{H} 2 \mathrm{AX}$ (Fig. 1a). However, some weak $\gamma \mathrm{H} 2 \mathrm{AX}$ foci are present on the axial element in zygotene and pachytene, indicating the presence of some (unrepaired) DSBs. The much lower intensity and limited presence of $\gamma \mathrm{H} 2 \mathrm{AX}$ (foci) on the GRC as compared to the rest of the nucleus indicates that only very few meiotic DSBs are induced on the heterochromatic GRC. At metaphase, an intense $\gamma \mathrm{H} 2 \mathrm{AX}$ signal covers the whole nucleus, including the relatively DAPI dense GRC (Fig. 2a).

When the nuclei progress from metaphase I through the first meiotic division and transform into secondary spermatocytes, the GRC appears to be eliminated from the nucleus, while its $\gamma \mathrm{H} 2 \mathrm{AX}$ signal increases strongly (Fig. 2a).

The GRC and its participation in meiotic recombination during spermatogenesis

To analyse the processing of meiotic DSBs on the GRC and the rest of the chromosomes in more detail, we immunostained for the homologous recombination repair protein RAD51. In leptotene spermatocytes, many small foci are present throughout the nucleus. During zygotene, the total number of RAD51 foci drops, and the remaining foci are larger and more intensely stained. In late zygotene, a few bright foci remain present, mainly on the unsynapsed parts of chromosome pairs. We also identified several RAD51 foci on the GRC, although the number and intensity of the foci is much less, and the foci itself appear smaller compared to the RAD51 foci on the other chromosomes (Fig. 2b).

A small fraction of the introduced meiotic DSBs are processed into crossovers. The DNA mismatch repair protein MLH1 marks the locations of these crossovers in spread meiotic nuclei of most species analysed to date. In mammals, MLH1 foci appear during midpachytene, and disappear before entering diplotene (Baker et al. 1996; Anderson et al. 1999). In chicken oocytes, MLH1 foci are already observed around mid to late zygotene (Pigozzi 2001).

In spermatocytes of the zebrafinch, many small MLH1 foci form aggregates around SYCP3 filaments of different chromosomes already at early zygotene. These aggregates appear to transform into bright MLH1 foci on the SCs around mid zygotene. Surprisingly, also the GRC shows a MLH1 focus in a minority of the nuclei (Fig. 2c), which provides an additional indication for the formation of meiotic DSBs in the GRC of some nuclei during leptotene. The MLHI foci on the autosomes remain present until diplotene, followed by dispersion of MLH1 signal throughout the nucleus towards the end of diplotene (not shown).

The GRC is silenced early during male meiotic prophase

The appearance of the DAPI dense GRC body during preleptotene indicates that it acquires a heterochromatic configuration upon entrance into meiotic prophase. To confirm this, we immunostained for a well-known marker of inactivation and heterochromatin, H3K9me3. We iden- 

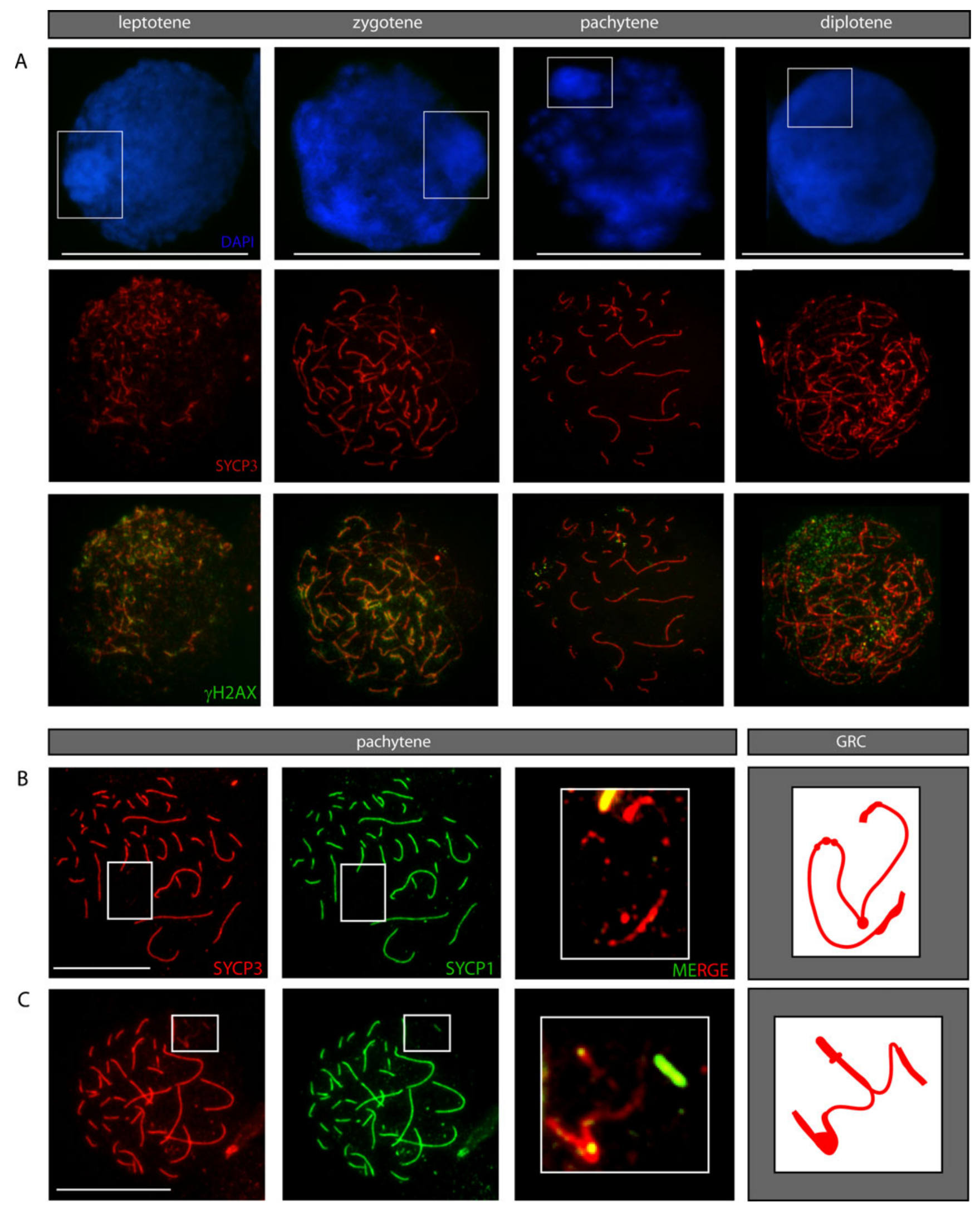
Fig. 1 The GRC throughout meiotic prophase in zebra finch spermatocytes. a Overview of the different stages of meiotic prophase I of zebra finch spermatocytes. The top panel shows the DAPI staining of the different nuclei, the lower panel shows the corresponding nuclei stained for SYCP3 (red), the bottom panel shows the same nuclei stained for both SYCP3 (red) and $\gamma \mathrm{H} 2 \mathrm{AX}$ (green). The position of the GRC is indicated by a box. Bar represents $10 \mu \mathrm{m}$. b, c Spermatocyte spread nuclei immunostained for SYCP3 (red) and SYCP1 (green). The GRC is boxed. Enlargement and the schematic drawing of GRC are shown. No (b) or a small fragment (c) of SYCP1 signal is present on the GRC. Bar represents $5 \mu \mathrm{m}$

tified preleptotene nuclei as the stage in which SYPC3 foci start to appear (Fig. 3a, b). Furthermore, the combined staining for SYPC3 and DAPI allowed us to distinguish between the different substages (early, mid and late) of preleptotene based on the appearance of a DDB (Fig. 3a-e). In the earlier preleptotene nuclei, we never observed a distinct subnuclear region containing such a strong H3K9me3 signal as was observed for the GRC body in leptotene spermatocyes (Fig. 3c, e). However, at the end of preleptotene, we observed colocalisation of the DDB and H3K9me3 (Fig 3c, e). For macroH2A, another heterochromatin marker, we observed a similar staining pattern as for $\mathrm{H} 3 \mathrm{~K} 9 \mathrm{me} 3$ (Figs. 3d and 7). This indicates that the GRC becomes heterochromatised upon entry into meiotic prophase.

The highly intense H3K9me3 staining, colocalising with the DDB remained present until its elimination. In metaphase I, H3K9me3 also marks the area of the centromeres (Fig. 3e).

We often observed a somewhat separate localisation of the GRC from the rest of the chromosomes around metaphase I, indicating that this chromosome may not be aligned with the rest of the chromosomes. In secondary spermatocytes, identified by the presence of a few SYPC3 filaments per nucleus (Fig. 4a), we observed nuclei with and without a
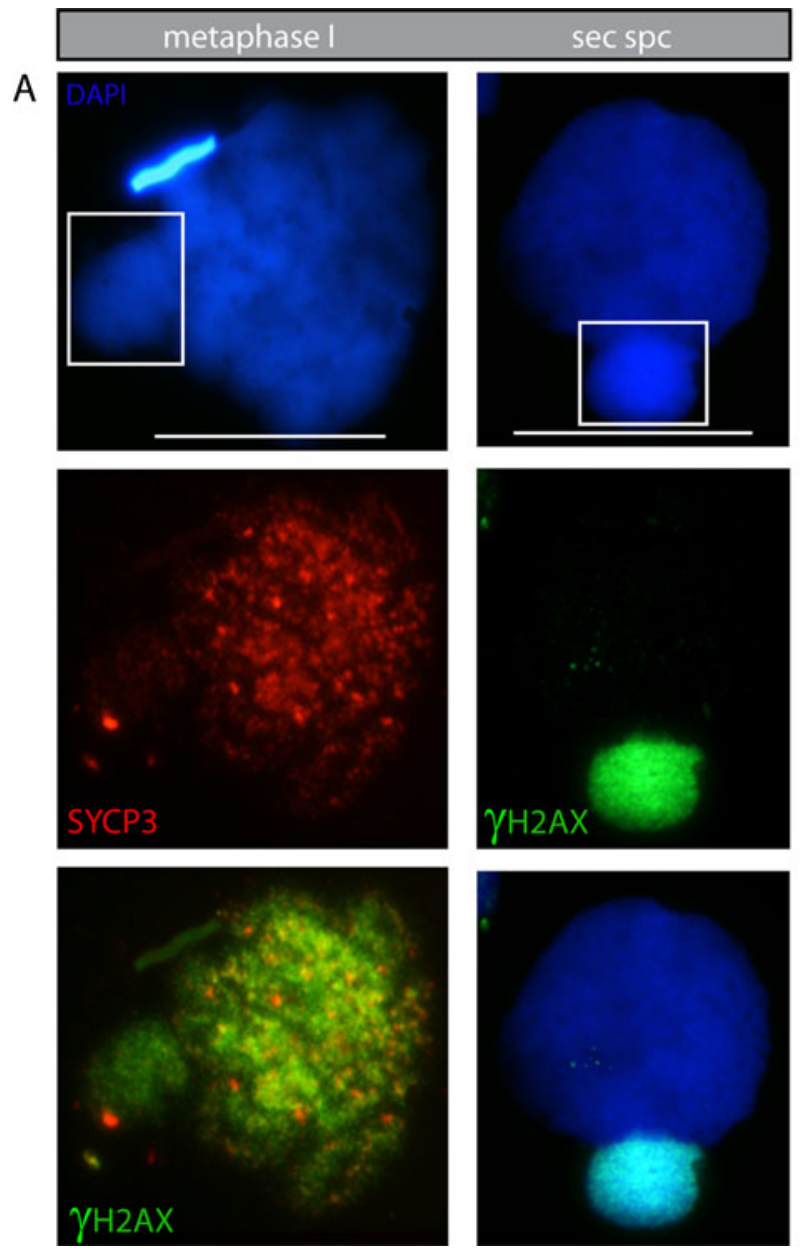

Fig. 2 The GRC and meiotic recombination. a Spread nuclei immunostained for DAPI (blue), $\gamma \mathrm{H} 2 \mathrm{AX}$ (green) and SYCP3 (red). After metaphase I, the GRC becomes strongly positive for $\gamma \mathrm{H} 2 \mathrm{AX}$. The GRC is boxed. Bar represents $5 \mu \mathrm{m}$. Sec spc secondary spermatocyte. b Spermatocyte spread nucleus immunostained for SYCP3 (red), RAD51 (green) and DAPI (blue). The GRC is boxed. Most RAD51 foci localise on axial elements of the SC. The bottom
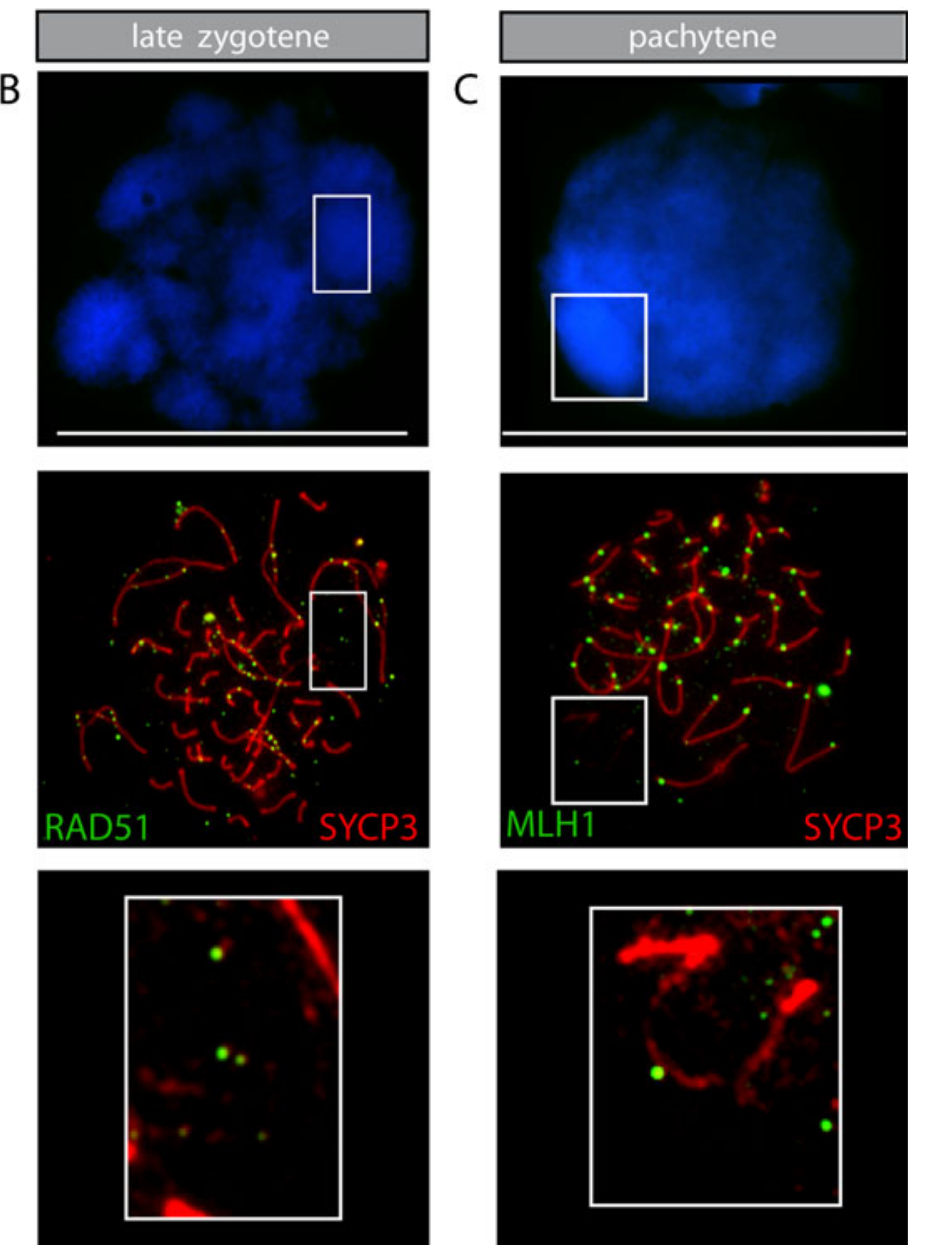

image represents a magnification of the area of the boxed GRC. Few small RAD51 foci are visible on the GRC. Bar represents $10 \mu \mathrm{m}$. c Spermatocyte spread nucleus immunostained for SYCP3 (red), MLH1 (green) and DAPI (blue). The area of the GRC is boxed. The bottom image represents a magnification of the area of the boxed GRC, and shows the presence of a MLH1 focus on the GRC. Bar represents $10 \mu \mathrm{m}$ 


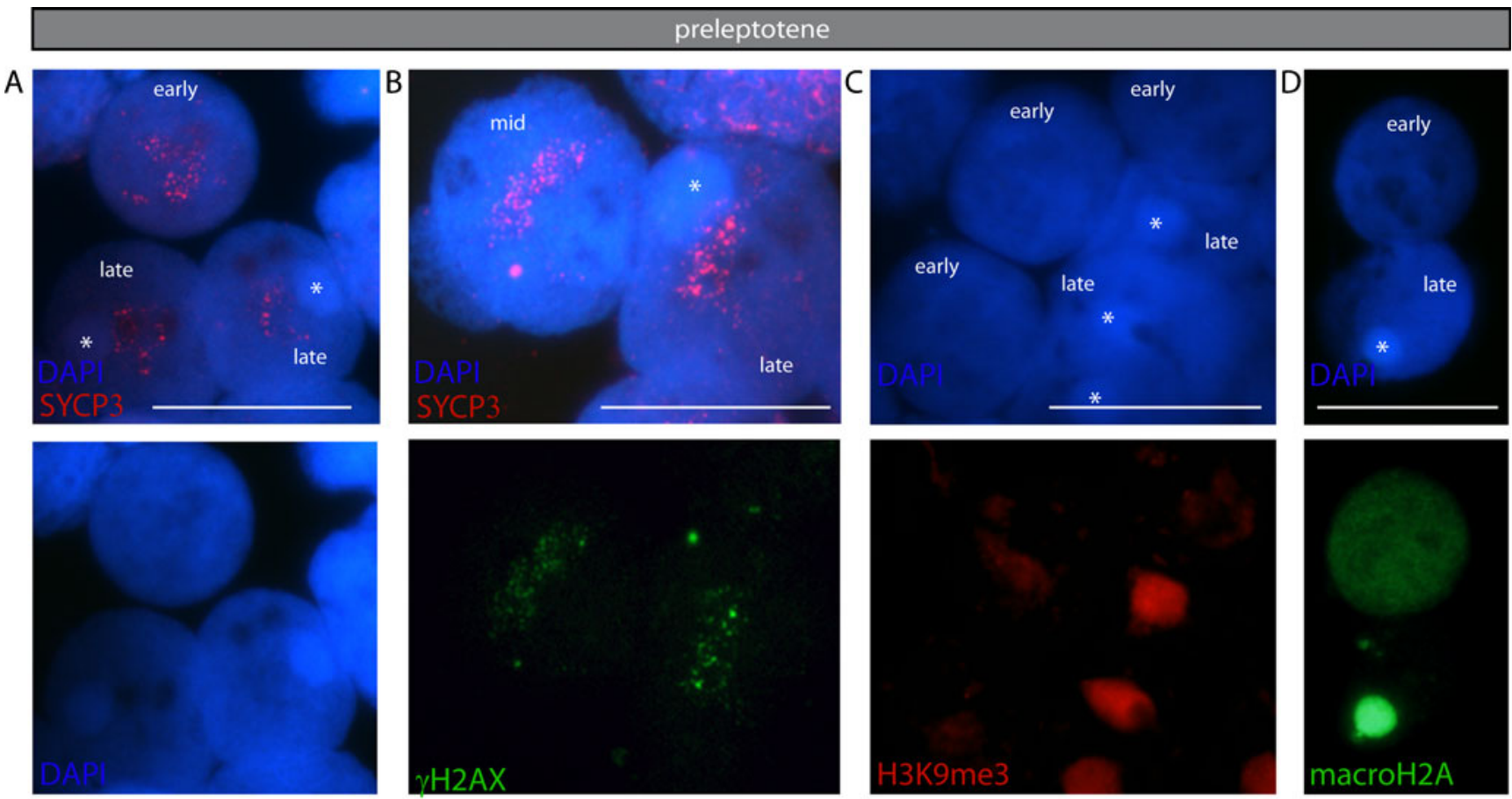

preleptotene

$\mathrm{E}$
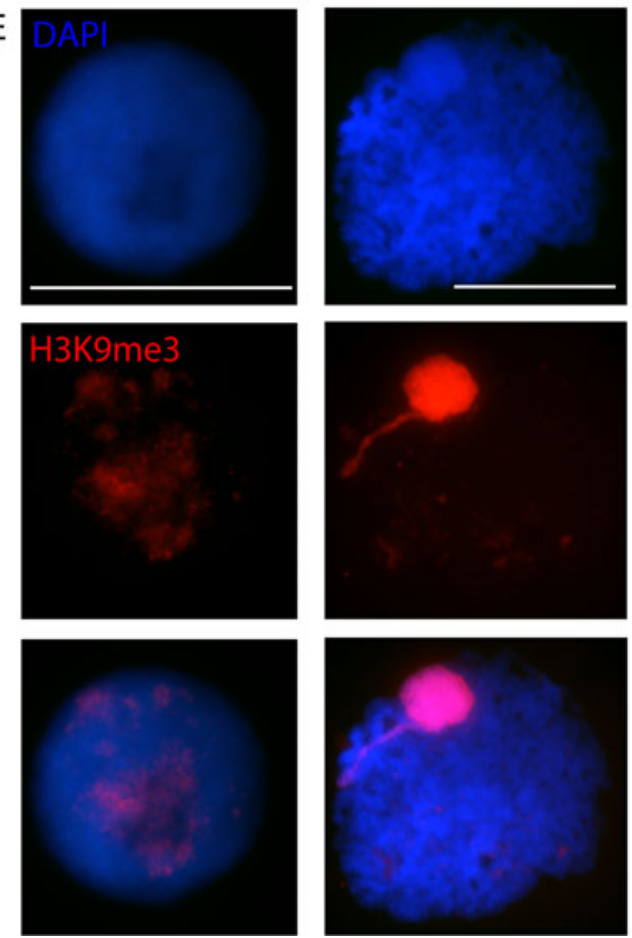

pachytene
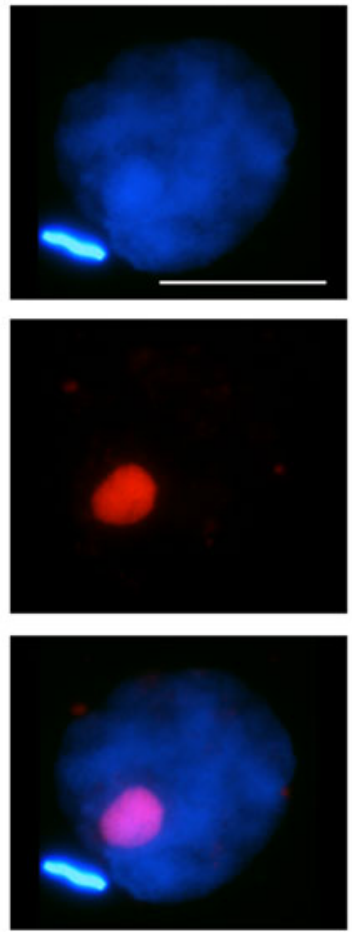

metaphase I
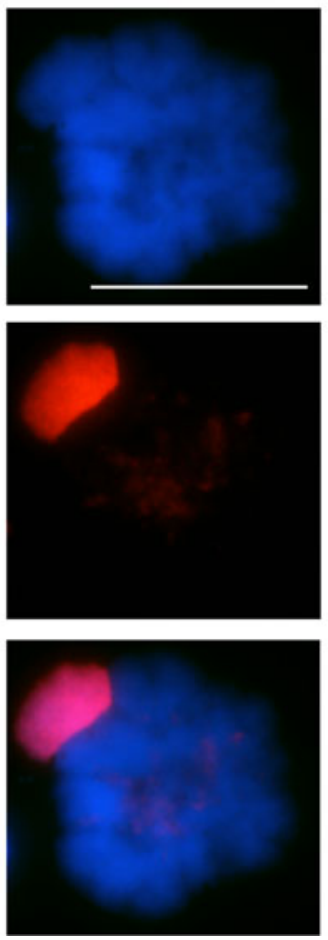

$\sec \operatorname{spc}$
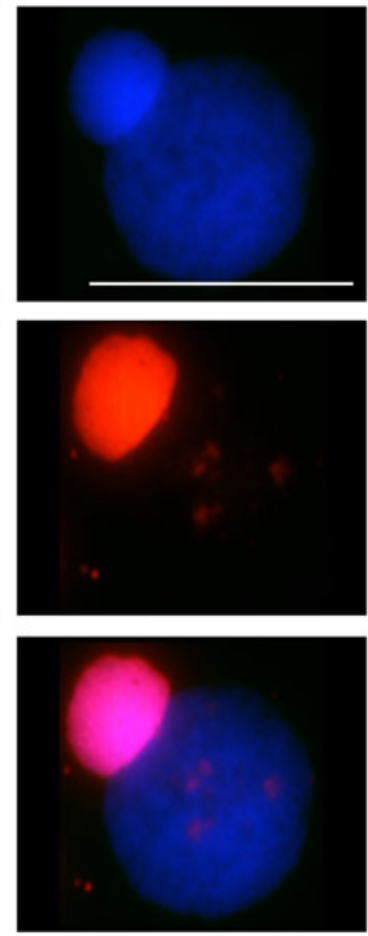

DAPI dense area, representing presence or absence of the GRC. To investigate this further, we quantified the number of nuclei without the GRC DAPI dense chromatin, nuclei with the GRC still minimally attached to the nucleus and nuclei still containing the GRC DDB (Fig. 4a). We scored 180 secondary spermatocyte nuclei and the vast majority
(131 out of 180 nuclei) had lost the GRC. A minority of the secondary spermatocytes appeared with a round, small DAPI dense GRC body still attached to the nucleus (36 out of 180 nuclei), and these bodies were still positive for H3K9me3 (Fig. 4a). We also observed secondary spermatocyte nuclei still containing an $\mathrm{H} 3 \mathrm{~K} 9 \mathrm{me} 3$ positive area, 
Fig. 3 The GRC is silenced during early meiotic prophase. a-d Preleptotene spermatocyte spread nuclei. Bar represents $5 \mu \mathrm{m}$. a Top panel shows early and late preleptotene nuclei stained for SYCP3 (red) and DAPI (blue). The DAPI dense GRC localisation in late preleptotene stages is indicated with an asterisk; the lower panel shows only the DAPI staining. The early preleptotene nucleus shows no heterochromatic area, indicating that the DDB has not yet formed. The formation of a few SYCP3 axial element fragments in all nuclei marks the preleptotene stage. b The top panel shows a mid and late preleptotene nucleus stained for SYCP3 (red) and DAPI (blue). The asterisk indicates the DAPI dense GRC. The lower panel shows the same nuclei, but immunostained for $\gamma \mathrm{H} 2 \mathrm{AX}$ (green), indicative of the presence of the first DNA double strand breaks that more or less colocalise with SYCP3. c, d The top panel shows early and late preleptotene stages with DAPI staining. The asterisk indicates the DAPI dense GRC. The lower panel shows the corresponding nuclei stained for $\mathrm{H} 3 \mathrm{~K} 9 \mathrm{me} 3$ (c) and macroH $2 \mathrm{~A}$ (d). Early preleptotene nuclei lack intense $\mathrm{H} 3 \mathrm{~K} 9 \mathrm{me} 3$ (c) and macroH2A (d) staining, and no DDB is observed. e The top panel shows DAPI staining of the different nuclei, the middle panel shows the corresponding nuclei stained for H3K9me3 (red) and the bottom panel shows the merge. In preleptotene, a faint staining is present throughout the nucleus. In all consecutive meiotic prophase stadia, the GRC is positive for H3K9me3. Sec spc secondary spermatocyte. Bar represents $5 \mu \mathrm{m}$

indicating that the GRC is not always eliminated at metaphase I (13 out of 180 nuclei; Fig. 3a).

We also immunostained for $\mathrm{H} 3 \mathrm{~K} 9 \mathrm{ac}$ and $\mathrm{H} 3 \mathrm{~K} 4 \mathrm{me} 3$ (Supplemental Fig. 1), as markers for active transcription. As expected, based on the staining for H3K9me3 and macroH2A, the GRC body lacked both markers of active chromatin from early leptotene until late diplotene. Whereas the GRC body remained devoid of H3K4me3, the H3K9ac signal on the DAPI dense GRC body, was no longer reduced compared to the rest of the chromatin from late diplotene onwards, indicating that the GRC acquires a mixed pattern of histone modifications at $\mathrm{H} 3 \mathrm{~K} 9$ around this stage.

\section{Analyses of known MSUC/MSCI markers} on the GRC body

Next, we investigated if markers of meiotic silencing of unsynapsed chromatin or more specifically, sex chromosomes (MSCI) also localise to the GRC body. The most well-known marker of the $\mathrm{XY}$ body in mammals is $\gamma \mathrm{H} 2 \mathrm{AX}$. However, as mentioned above, $\gamma \mathrm{H} 2 \mathrm{AX}$ is mostly absent from the GRC until metaphase. Still, $\gamma \mathrm{H} 2 \mathrm{AX}$ starts to mark the GRC body from metaphase onwards, and the GRC remains $\gamma \mathrm{H} 2 \mathrm{AX}$ positive until it is expelled from the nucleus (Fig. 2a).

H2AK119 ubiquitylation, another XY and ZW body marker, also marked the GRC, starting just after metaphase I and reaching its maximum in secondary spermatocytes, when it is still attached to the nucleus (Fig. 4b). Free GRC bodies were mostly negative for H2AK119 ubiquitylation (not shown).

In mouse (Vigodner and Morris 2005; Vigodner 2009), the $\mathrm{XY}$ body is marked by increased protein sumoylation from late zygotene onwards, whereas the $\mathrm{XY}$ body in human (Metzler-Guillemain et al. 2008) and the ZW body in chicken (not shown) show no increased sumoylation. We found strong staining for sumoylation on the GCR body from leptotene onwards, and the signal remained present until late pachytene (Fig. 4c).

Lack of H4K16ac marks the inactive $\mathrm{ZW}$ pair at during pachytene in chicken oocytes (Schoenmakers et al. 2009). However, we observed marked H4K16ac staining, colocalising with the GRC body during leptotene, zygotene and reaching a maximum at pachytene (Fig. 4d). From metaphase onwards, H4K16ac staining was evenly distributed throughout the nucleus, including the GRC body (not shown).

\section{Absence of INCENP from the GRC}

During metaphase I, attachment of spindle microtubules to the kinetochores enables segregation of dyads during the first meiotic division.

We found that the GRC mostly localises to the periphery of the nucleus during meiotic prophase. In metaphase I, we noticed that the GRC often appears separate from the other chromosomes. Earlier reports also described that the GRC becomes separate from the rest of the DNA during metaphase I (Pigozzi and Solari 1998; Pigozzi and Solari 2005). The distant position of the GRC and its apparent separation could be caused by defective or absent attachment of the GRC to the spindle. To analyse this, we immunostained for the centromeric protein inner centromere protein (INCENP). During male meiosis in mouse, INCENP localises on the central elements of the synaptonemal complex from zygotene onwards. In late pachytene, it relocalises to the centromeres (Parra et al. 2009). In zebra finch spermatocytes, we first observed the appearance of INCENP on the SC fragments around late zygotene-early pachytene and as bright foci throughout the nuclei (Fig. 5a). However, the area containing the GRC lacked INCENP staining. Around metaphase I, most of the INCENP foci disappeared and the protein relocalised to the centre of the nucleus. During progression of metaphase I, we observed intense INCENP staining indicating the positions of the centromeres. In the vast majority of the nuclei, the GRC lacked all INCENP signals. However, rarely, the GRC did show INCENP staining, which could indicate that the GRC participates in the first meiotic division in a small minority of the cells (not shown).

DNA fragmentation of the GRC

during and after its elimination from the nucleus

Both phosphorylation of $\mathrm{H} 2 \mathrm{AX}$, and ubiquitylation/deubiquitylation of $\mathrm{H} 2 \mathrm{~A}$ have been associated not only with 


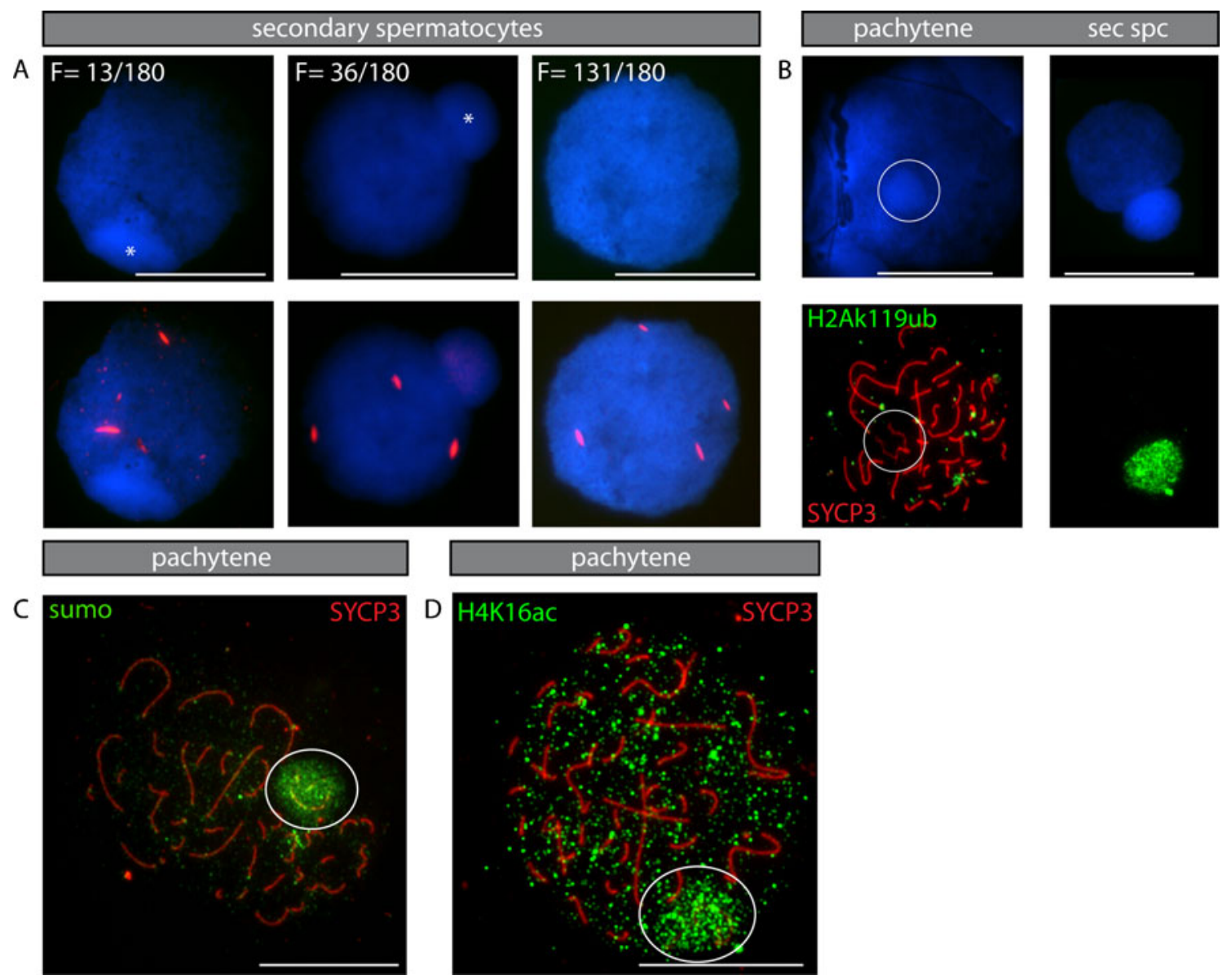

Fig. 4 The silenced GRC is eliminated at metaphase I in the vast majority of spermatocytes. a Spermatocyte spread nuclei stained for DAPI (blue, upper and lower panels) and SYCP3 (lower panel, red). The left image shows a secondary spermatocyte with the GRC still inside the nucleus, the middle image with the GRC still attached to the outside of the nucleus and the right image shows a secondary spermatocyte lacking the GRC. The typical SYCP3 filaments/dots mark the secondary spermatocyte stage. $F$ the number of positive nuclei/the total number of scored secondary spermatocyte nuclei. Bar

DSB repair and chromatin silencing, but also with initiation and execution of apoptosis, which involves the generation of DNA breaks and finally results in DNA fragmentation (Rogakou et al. 2000; Mimnaugh et al. 2001; Sluss and Davis 2006; Zhou et al. 2009). The appearance of increasing $\gamma \mathrm{H} 2 \mathrm{AX}$ and H2AK119 ubiquitylation staining on the GRC from metaphase I onwards (Figs. 2a and 4b), combined with initial lack of $\gamma \mathrm{H} 2 \mathrm{AX}$ and H2AK119 ubiquitylation staining during pachytene, indicated that the DNA of the GRC may be degraded during and after its release from the nucleus. Terminal deoxynucleotidyl transferase dUTP nick end labelling (TUNEL) staining is a common method used for the detection of DNA fragmentation. We observed faint TUNEL staining in early meiotic prophase spermatocytes, which most likely represents the presence of meiotic DSBs. The GRC body showed less represents $5 \mu \mathrm{m}$. b, $\mathbf{c}, \mathbf{d}$ Male meiotic prophase spread nuclei stained for H2AK119 ubiquitylation (green, b), sumoylation (green, c), H4K16ac (green, d) and SYCP3 (red). At pachytene, the GRC becomes increasingly sumoylated, and acetylated on H4K16. However, no H2AK119 ubiquitylation signal is present on the GRC at this stage. When the GRC is almost eliminated, it becomes increasingly ubiquitylated at H2AK119 (b). The area of the GRC is encircled. Bar represents $5 \mu \mathrm{m}$

TUNEL staining compared to the rest of the chromosomes during these stages, which is in agreement with the relative lack of RAD51 and $\gamma \mathrm{H} 2 \mathrm{AX}$ foci on the GRC we observed previously. When the GRC body becomes more separate from the rest of the chromosomes, TUNEL signal increases, and when the GRC has formed a separate body, the TUNEL staining is maximal, again indicating that the DNA of the GRC is fragmented during and after its exclusion from the nucleus (Fig. 5b).

Analyses of the GRC during female meiotic prophase of the zebra finch

To compare the behaviour of the single GRC in male spermatocytes with that of the paired GRC and the ZW chromosome pair in oocytes, we immunostained oocyte 

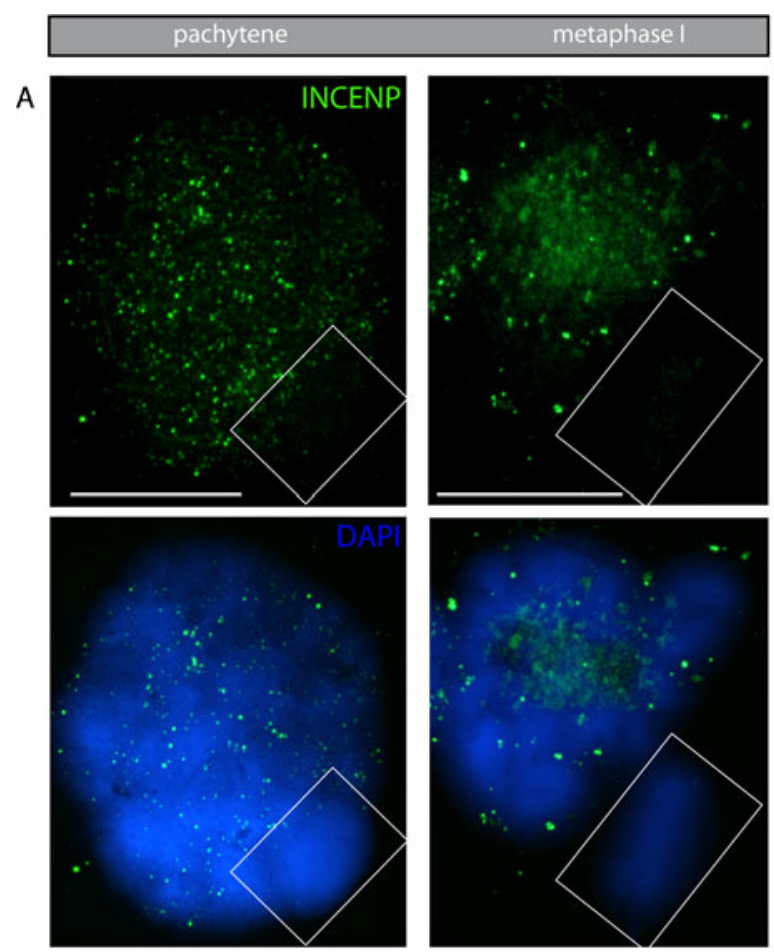

Fig. 5 The GRC lacks INCENP protein, and its DNA is degraded. a Male meiotic prophase spread nuclei stained for INCENP (green) and DAPI (blue). In pachytene, INCENP foci most likely follow the axial elements, which can be inferred from the DAPI staining. Also, many INCENP foci are present throughout the nucleus. The GRC remains largely devoid of INCENP staining. From diplotene onward, INCENP foci relocalise and concentrate mainly around the centromeres in

spread preparations of zebra finch females for SYCP3 and H3K9me3 and for SYCP3 and H2AK119 ubiquitylation (Fig. 6).

As we observed previously in chicken oocytes (Schoenmakers et al. 2009), a H3K9me3-positive ZW body is visible during pachytene in zebra finch oocytes. H2AK119 ubiquitylation also marks part of the ZW pair around mid pachytene. In addition, we observed that the middle part of the GRC pair is also positive for $\mathrm{H} 3 \mathrm{~K} 9 \mathrm{me} 3$, shows a dense DAPI signal, and mostly localises in the periphery of the nucleus. Throughout the female meiotic prophase, the GRCs remain partially positive for $\mathrm{H} 3 \mathrm{~K} 9 \mathrm{me} 3$ (Fig. 6), although the signal is much less intense compared to what we observed for the single GRC in spermatocytes. In pachytene and diplotene, part of the GRC shows a very strong $\mathrm{H} 3 \mathrm{~K} 9 \mathrm{me} 3$ signal and this part of the GRC often colocalises with the $\mathrm{H} 3 \mathrm{~K} 9 \mathrm{me} 3$ positive $\mathrm{ZW}$ pair in the periphery of the nucleus.

\section{Discussion}

During male and female meiotic prophase in mammals, the presence of unsynapsed chromatin is associated with silencing

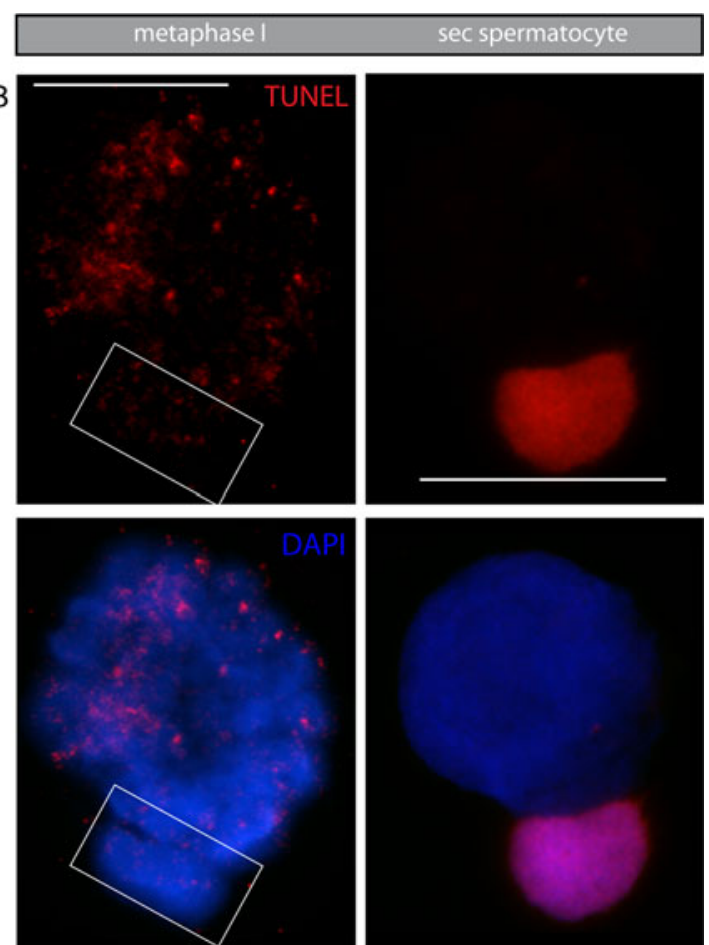

metaphase. The GRC mostly shows no INCENP staining at its centromere. The area of the GRC is boxed. Bar represents $5 \mu \mathrm{m}$. b Spermatocyte spread nuclei stained for TUNEL (red) and DAPI (blue). Early in meiosis the GRC is largely devoid of TUNEL staining. When the GRC is almost expelled from the nucleus, it becomes strongly TUNEL-positive. The area of the GRC is boxed. Bar represents $5 \mu \mathrm{m}$

of these areas by MSUC. The heterologous XY pair remains largely unsynapsed, and is always subjected to a specialised form of MSUC: meiotic sex chromosome inactivation (reviewed in Turner 2007). In mammals, MSUC and MSCI appear to be triggered by the presence of unsynapsed chromatin in combination with persistent meiotic DSBs (Turner 2007; Schoenmakers et al. 2008). Recently, we showed that the avian sex chromosomes in chicken oocytes also display MSCI, albeit transient, despite the fact that $\mathrm{Z}$ and W show complete synapsis (Schoenmakers et al. 2009). Here, we asked how, in addition to the avian form of MSCI, meiotic silencing of unsynapsed chromatin functions in the avian germline. We chose to analyse the meiotic behaviour of the single copy of the GRC in the germline of the male zebrafinch. Our data show that this chromosome is silenced upon entry into meiotic prophase, first detectable in preleptotene spermatocytes. This resembles what we observed for the $\mathrm{W}$ chromosome in chicken and zebra finch oocytes. Apparently, the inactivation of both the GRC and W chromosomes precedes the introduction of meiotic DSBs and synapsis. Furthermore, the GRC does appear to accumulate only few meiotic DSBs during leptotene. Therefore, it is unlikely that the mechanisms that silence $\mathrm{W}$ and the GRC are linked to meiotic DSB repair. In addition, 


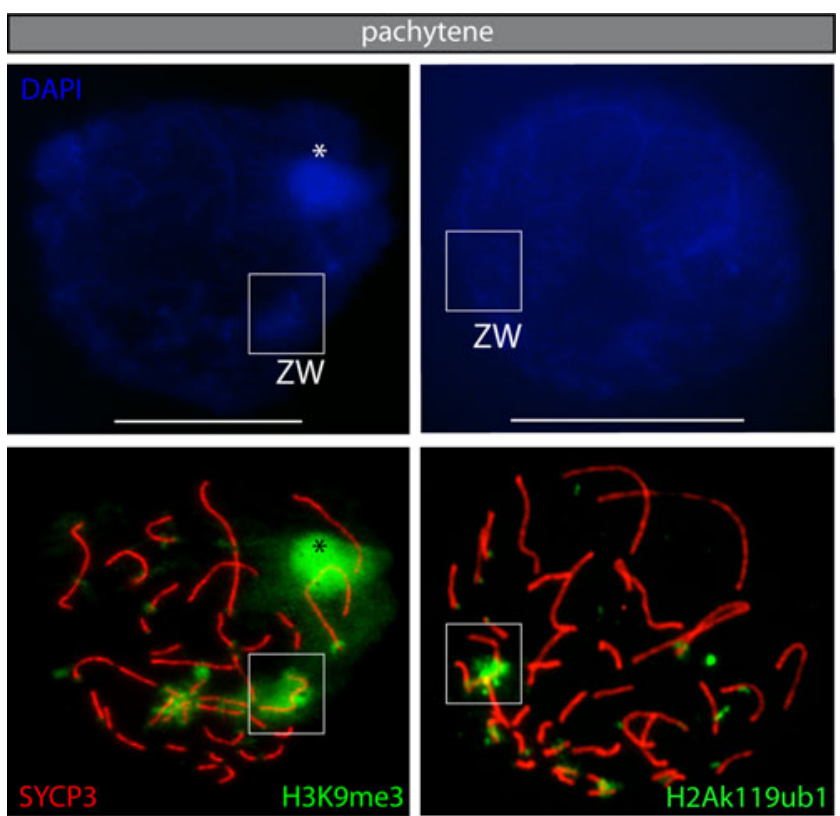

Fig. 6 Analyses of the ZW pair and GRC chromosomes during female meiotic prophase. Oocyte spread nuclei stained for SYCP3 (red), H3K9me3 (green), H2AK119ub (green) and DAPI (blue). At pachytene, the $\mathrm{ZW}$ pair is strongly positive for $\mathrm{H} 3 \mathrm{~K} 9 \mathrm{me} 3$ and H2Ak119ubiquitylation. Asterisk indicates the middle part of the GRC, which is also positive for $\mathrm{H} 3 \mathrm{~K} 9 \mathrm{me} 3$, indicating that the GRC is also (partially) silenced during female meiotic prophase. GRC is boxed. Bar represents $5 \mu \mathrm{m}$

since silencing of W (in oocytes) and the GRC (in spermatocytes) occurs prior to initiation of chromosome pairing, discrimination between synapsis/asynapsis can also not be the initiating trigger for this transcriptional silencing (Fig. 7).

In male grasshoppers, Eyprepocnemis plorans and Locusta migratoria, the single facultative heterochromatic $\mathrm{X}$ chromosome (and a constitutive heterochromatic B chromosome) remain unsynapsed throughout meiotic prophase and are also silenced at the onset of meiotic prophase (Viera et al. 2004a; b) as visualised by the loss of H3K9 acetylation (Cabrero et al. 2007; Viera et al. 2004a). Apparently, in spermatocytes of grasshoppers, silencing of these single chromosomes is also DSB and synapsisindependent. Based on these observations, we would like to propose to refer to this type of early meiotic silencing of chromosomes as meiotic silencing prior to synapsis (MSPS), to indicate that the initiating events and timing in this type of meiotic silencing may be different from MSUC in mammals.

Cabrero et al. (2007) suggested the existence of a counting mechanism operating at the start of meiosis, enabling detection and direct subsequent inactivation of single chromosomes. The clustering of telomeres in the nuclear envelope that occurs upon entry in meiotic prophase I (reviewed in Scherthan 2007) could play a role in the detection of these univalents. For zebra finch and chicken, such a counting mechanism seems unlikely, since the single $\mathrm{Z}$ chromosome behaves different from the single $\mathrm{W}$ and GRC.

$\mathrm{W}$ and the GRC appear euchromatic in premeiotic germ cell stages, but may be largely transcriptionally silent due to the fact that very few functional genes appear to be present. In fact, these chromosomes seem to be highly enriched for repeat sequences (Itoh et al. 2009), and their inactive status may be associated with late replication during the final $\mathrm{S}$ phase before entry into meiotic prophase. Recently, LandeDiner et al. (2009) showed that replication-time switching, from late to early or vice versa, is linked to the acetylation state of the chromatin, and thus may direct nucleosome structure regulation. Perhaps the nucleosomes that assemble on GRC and $\mathrm{W}$ chromatin during the final $\mathrm{S}$ phase are specifically modified, for example by acetylation of H4K16, to mark them for global silencing and heterochromatisation, and, in case of the GRC, to prepare it for its ultimate expulsion. Apart from, or in addition to, late replication, small non-coding RNA pathways may also be involved, since these have been linked to silencing of transposable elements and repeats in meiosis. The elimination of the GRC during male meiosis shows some resemblance to homology-dependent programmed (single copy) DNA elimination that is also mediated by non-coding RNAs during sexual reproduction in ciliates (reviewed in Bernstein and Allis 2005; Lepere et al. 2008; Mochizuki and Gorovsky 2004) .

Surprisingly, we found that in contrast to what has been described for the grasshopper $\mathrm{X}$ and $\mathrm{B}$ chromosomes, the GRC occasionally accumulates a few meiotic DSBs, as shown by the presence of both RAD51 and $\gamma \mathrm{H} 2 \mathrm{AX}$ foci. Its compact heterochromatic configuration most likely prevents access of DSB-inducing proteins to most of the GRC, minimising the number of DSBs in the GRC. Rarely, we noticed the presence of a MLH1 focus on the GRC. The existence of intrachromosomal recombination could explain this observation, and this is supported by the recent finding that the GRC is rich in repeat sequences (Itoh et al. 2009) and our observation of small regions of self-synapsis, visualised by the presence of small SYCP1 stretches on the GRC during pachytene.

H4K16 acetylation is generally associated with an open chromatin configuration and a transcriptionally active state (reviewed in Shahbazian and Grunstein 2007). However, we find increased H4K16 acetylation on the heterochromatic GRC. Although there are a few reports describing that histone acetyltransferases could be involved in maintaining silencing of certain loci, this most likely results from shifting of heterochromatic boundaries and not from direct silencing activity induced by the histone acetylation (reviewed in Shahbazian and Grunstein 2007). Therefore, it is unlikely that the observed increased H4K16 acetylation is linked to GRC silencing. Histone acetyltransferases have also been implicated in the detection of DSBs and DNA 

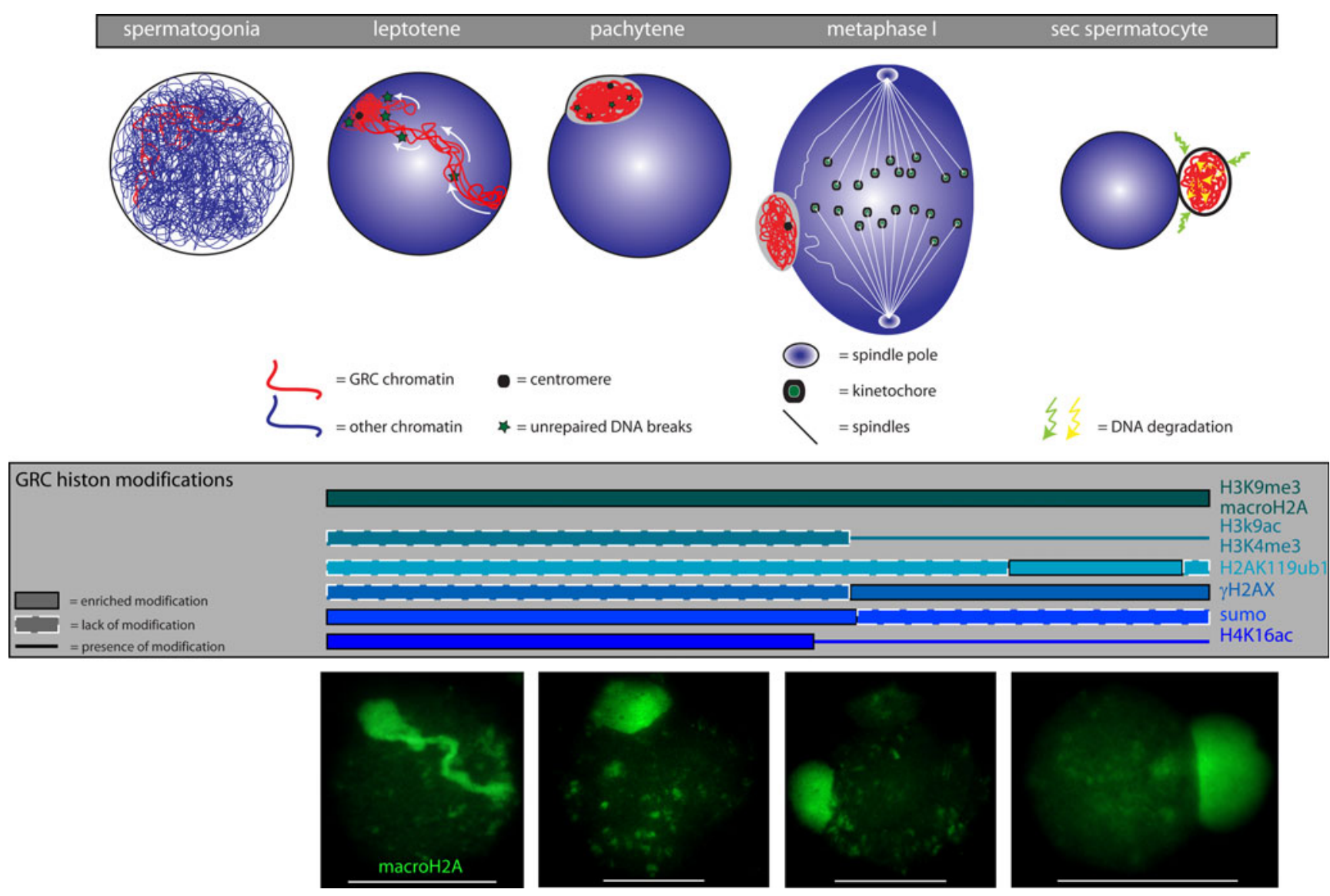

Fig. 7 Schematic overview of the behaviour of the GRC during male meiosis of the zebra finch. In the transition phase between spermatogonia and the start of the male meiotic prophase, the GRC is identified and from preleptotene, it becomes heterochromatised and gains specific chromatin modifications such as $\mathrm{H} 3 \mathrm{~K} 9 \mathrm{me} 3$, macroH2A, sumoylation and H4K16ac. With progression through meiotic prophase, all chromatin of the GRC is drawn into a heterochromatic body at the periphery of the nucleus. Few DSBs are induced on the GRC, and these remain unrepaired. Also, H2A on its centromere is not ubiquitylated. Sumoylation of chromatin and acetylation at H4K16 accumulate on the GRC and reach a maximum around mid pachytene. At diplotene, the GRC becomes acetylated at $\mathrm{H} 3 \mathrm{~K} 9$ and starts to decondense. During metaphase I, the nuclear membrane is degraded, the centromere and kinetochore of the GRC are aberrant and proper attachment to the spindles fails. This may cause DNA degradation of the GRC, and the chromatin to become phosphorylated, ubiquitylated and again de-ubiquitylated. In addition, the GRC forms a micronucleus with ongoing DNA fragmentation in the cytoplasm of the secondary spermatocyte

and ensures correct kinetochore-microtubule interactions (reviewed in Vagnarelli and Earnshaw 2004). The lack of INCENP loading on the GRC indicates that the separation of the GRC from the rest of the chromosomes around metaphase $\mathrm{I}$ is at least partly due to the fact that proper microtubule attachment to the GRC kinetochore may not occur, resulting in aberrant localisation of the chromosome. In addition, the fact that the GRC is not attached to a homolog will contribute to its aberrant behaviour; the lack of tension from opposing pulling forces will destabilise the attached microtubules. Abberant regulation of the kinetochore/centromere is also apparent from the lack of H2AK119 ubiquitylation (not shown), which marks the centromeres on other chromosomes. Around the first meiotic metaphase-toanaphase transition, the GRC is truly separated from the two haploid genomes and will no longer be incorporated in the
CENP, is part of the conserved chromosomal passer ger complex, which regulates chromosomal segregation 
nucleus in the vast majority of secondary spermatocytes. Instead, the GRC forms a micronucleus.

When the GRC does not move to the metaphase plate, DNA fragmentation that selectively affects the GRC could be triggered, as visualised by increasing $\gamma \mathrm{H} 2 \mathrm{AX}, \mathrm{H} 2 \mathrm{AK} 119$ ubiquitylation and TUNEL staining. Subsequently, deubiquitylation of $\mathrm{H} 2 \mathrm{~A}$ follows, which is a feature of ongoing apoptosis (Mimnaugh et al. 2001). These events are schematically summarised in Fig. 7. Recently, Terradas et al. (2009) showed that also in human cells, DNA with unrepaired DSBs can be separated in a micronucleus, in which DNA degradation occurs, followed by elimination. Elimination of the single GRC may have evolved to avoid activation of the spindle checkpoint. For example, in mantid spermatocytes, sex chromosomes occasionally remain unpaired, and this leads to phosphorylation of the kinetochore, checkpoint activation and meiotic arrest ( $\mathrm{Li}$ and Nicklas 1997). In contrast, in XO grasshopper species, the always single $\mathrm{X}$ has a special kinetochore that apparently is not recognised by the checkpoint machinery ( $\mathrm{Li}$ and Nicklas 1997).

In the female germline, the GRC is present as a bivalent, in most oocytes. It can be easily recognised because it is the longest bivalent (Pigozzi and Solari 1998; Pigozzi and Solari 2005). We noticed that it is also partly heterochromatic, which may be caused by a low gene content and/or the presence of repetitive DNA on these chromosomes. In chicken oocytes, we have also observed increased H3K9me3 staining on parts of the macrochromosomes (Schoenmakers et al. 2009). In contrast to the single GRC in males, the GRC bivalent in oocytes normally participates in meiotic recombination. It has been suggested that recombination of the GRC pair in the female germline prevents the loss of the GRC and ensures the transmission through females (Pigozzi and Solari 2005). From this perspective, recombination of $Z$ with $\mathrm{W}$ in the pseudoautosomal region may rescue $\mathrm{W}$ from a fate similar to that of the single GRC in males. Alternatively, failure of pairing leading to a single $\mathrm{W}$ may trigger meiotic arrest. A special feature intrinsic to the GRC may be responsible for the aberrant kinetochore/centromere structure, causing initiation of the DNA fragmentation and elimination from the male meiotic nucleus.

We have shown that the single unpaired GRC in male zebra finch meiosis is efficiently silenced, and propose to refer to this phenomenon as MSPS. The timing of meiotic silencing in birds differs from that of meiotic silencing in mammals: Whereas the GRC and $\mathrm{W}$ are inactivated immediately upon entry into meiotic prophase, MSUC and MSCI in mammals is initiated at the zygotene/pachytene transition, strictly correlating with the timing of an unsynapsed configuration. In birds and mammals, essentially different machineries may have evolved to trigger meiotic silencing of regions without a pairing partner.

\section{Materials and methods}

Spreads and immunocytochemistry

Adult male and 2-7-day-old female zebra finches (T. guttata) were killed by $\mathrm{CO}_{2}$ intoxication. The functional left ovary and left and right testes were dissected and placed in PBS solution. Spread nuclei preparations of zebra finch oocytes and spermatocytes were prepared using a modification of the drying-down technique described by Peters et al. (1997). Briefly, ovaries and testes were minced with forceps, and ovarian cells were suspended in $500 \mu \mathrm{l}$ of $100 \mathrm{mM}$ sucrose, containing EDTA-free complete protease inhibitor cocktail (Roche Diagnostics, Almere, The Netherlands), whereas testicular cells were placed in hypobuffer solution, and after centrifugation, cells were resuspended in $100 \mathrm{mM}$ sucrose. Oocytes and spermatocytes were dispersed on a glass slide dipped in $1 \%$ paraformaldehyde fixative with $0.1 \%$ Triton X100. After $2 \mathrm{~h}$ in a humid chamber at room temperature, the slides were allowed to dry for 30-60 $\mathrm{min}$ at room temperature, followed by a single wash in $0.08 \%$ Photoflo (Kodak, Paris, France) and air-dried. The slides were stored at $-80^{\circ} \mathrm{C}$.

For immunocytochemistry, frozen slides were defrosted at room temperature and washed with PBS. The slides were blocked with PBS containing $0.5 \% w / v$ BSA and $0.5 \% w / v$ milk powder, and double stained with different combinations of the following antibodies: rabbit polyclonal anti-SYCP3 $(1: 1,000)$, rabbit polyclonal anti-SYCP1 (1:200; gifts from C. Heyting, Wageningen), mouse polyclonal anti- $\gamma \mathrm{H} 2 \mathrm{AX}$ (1:1,000; Upstate, Walthum, MA, USA), mouse monoclonal IgM anti-H2AK119ub1 (1:1,000; Upstate), mouse monoclonal anti-H4K16ac (1:200; Upstate), mouse monoclonal MLH1 (1:25; BD Pharmingen, San Diego, USA), rabbit polyclonal anti-H3K9me3 (1:500; Upstate), rabbit antihuman RAD51 (1:500) [24], rabbit polyclonal macroH2A (1:500; Upstate), rabbit monoclonal H3K9ac (1:50; Sigma, USA), H3K4me3 (1:1,000; Upstate), mouse monoclonal GMP-1 (SUMO; 1:100; Zymed, San Francisco, USA) and rabbit polyclonal INCENP (1:100; Abcam, UK). For mouse monoclonal primary antibodies, the secondary antibodies were fluorescein isothiocyanate (FITC; 1:128; Sigma, St. Louis, USA)-labelled goat anti-mouse IgG antibodies for anti- $\gamma \mathrm{H} 2 \mathrm{AX}$, anti-MLH1, anti-macroH2A and anti-GMP1, FITC-labelled goat anti-mouse IgM (1:128; Sigma) for anti-H2AK119ub1 and tetramethylrhodamine isothiocyanate (1:128; Sigma)-labelled goat anti-mouse IgG antibodies for anti- $\gamma \mathrm{H} 2 \mathrm{AX}$. The secondary antibody for polyclonal rabbit primary antibodies was tetramethylrhodamine isothiocyanate (1:200; Sigma)-labelled goat anti-rabbit IgG antibodies for anti-SYCP3 and fluorescein isothiocyanate (1:80; Sigma)labelled goat anti-rabbit IgG antibodies for anti-RAD51, antiSYCP1, anti-H3K9ac and anti-INCENP. Primary antibodies 
were diluted in $10 \% w / v$ BSA in PBS and incubated overnight in a humid chamber. Thereafter, slides were washed in PBS, blocked in $10 \% v / v$ normal goat serum (Sigma) in blocking buffer $(5 \%$ milk powder $(w / v)$ in PBS, centrifuged at 13,200 rpm for $10 \mathrm{~min}$ ), and incubated with secondary antibodies in $10 \% v / v$ normal goat serum in blocking buffer at room temperature for $2 \mathrm{~h}$. Next, the slides were washed in PBS and embedded in Vectashield containing 4', $6^{\prime}$ diamindino-2-phenylindole (Vector Laboratories, Burlingame CA, USA). Double stainings of SYCP1 with SYCP3 and of RAD51 with SYCP3 (all rabbit polyclonal antibodies) were obtained by sequential immunostainings with the single antibodies. Images of SYCP1, RAD51 and SYCP3 stainings, respectively, were obtained prior to immunostaining with antiSYCP3 of the same nuclei.

Acknowledgements We want to thank Prof. Dr. Simon Verhulst and Egbert Koetsier of the University of Groningen for advice and technical assistance. This work was supported by the Netherlands Organisation for Scientific Research (NWO) through ALW (VIDI 864.05.003).

Open Access This article is distributed under the terms of the Creative Commons Attribution Noncommercial License which permits any noncommercial use, distribution, and reproduction in any medium, provided the original author(s) and source are credited.

\section{References}

Anderson LK, Reeves A, Webb LM, Ashley T (1999) Distribution of crossing over on mouse synaptonemal complexes using immunofluorescent localization of MLH1 protein. Genetics 151:1569-1579

Ashley T, Plug AW, Xu J, Solari AJ, Reddy G, Golub EI, Ward DC (1995) Dynamic changes in Rad51 distribution on chromatin during meiosis in male and female vertebrates. Chromosoma 104:19-28

Baker SM, Plug AW, Prolla TA, Bronner CE, Harris AC et al (1996) Involvement of mouse Mlh1 in DNA mismatch repair and meiotic crossing over. Nat Genet 13:336-342

Bernstein E, Allis CD (2005) RNA meets chromatin. Genes Dev 19:1635-1655

Cabrero J, Teruel M, Carmona FD, Jimenez R, Camacho JP (2007) Histone $\mathrm{H} 3$ lysine 9 acetylation pattern suggests that $\mathrm{X}$ and $\mathrm{B}$ chromosomes are silenced during entire male meiosis in a grasshopper. Cytogenet Genome Res 119:135-142

Itoh Y, Kampf K, Pigozzi MI, Arnold AP (2009) Molecular cloning and characterization of the germline-restricted chromosome sequence in the zebra finch. Chromosoma 118:527-536

Keeney S, Giroux CN, Kleckner N (1997) Meiosis-specific DNA double-strand breaks are catalyzed by Spo11, a member of a widely conserved protein family. Cell 88:375-384

Lande-Diner L, Zhang J, Cedar H (2009) Shifts in replication timing actively affect histone acetylation during nucleosome reassembly. Mol Cell 34:767-774

Lepere G, Betermier M, Meyer E, Duharcourt S (2008) Maternal noncoding transcripts antagonize the targeting of DNA elimination by scanRNAs in Paramecium tetraurelia. Genes Dev 22:15011512

Li X, Nicklas RB (1997) Tension-sensitive kinetochore phosphorylation and the chromosome distribution checkpoint in praying mantid spermatocytes. J Cell Sci 110(Pt 5):537-545
Mahadevaiah SK, Turner JM, Baudat F, Rogakou EP, de Boer P, Blanco-Rodriguez J, Jasin M, Keeney S, Bonner WM, Burgoyne PS (2001) Recombinational DNA double-strand breaks in mice precede synapsis. Nat Genet 27:271-276

Metzler-Guillemain C, Depetris D, Luciani JJ, Mignon-Ravix C, Mitchell MJ, Mattei MG (2008) In human pachytene spermatocytes, SUMO protein is restricted to the constitutive heterochromatin. Chromosome Res 16:761-782

Mimnaugh EG, Kayastha G, McGovern NB, Hwang SG, Marcu MG, Trepel J, Cai SY, Marchesi VT, Neckers L (2001) Caspasedependent deubiquitination of monoubiquitinated nucleosomal histone H2A induced by diverse apoptogenic stimuli. Cell Death Differ 8:1182-1196

Mochizuki K, Gorovsky MA (2004) Small RNAs in genome rearrangement in Tetrahymena. Curr Opin Genet Dev 14:181-187

Monesi V (1965) Differential rate of ribonucleic acid synthesis in the autosomes and sex chromosomes during male meiosis in the mouse. Chromosoma 17:11-21

Moens PB, Chen DJ, Shen Z, Kolas N, Tarsounas M, Heng HH, Spyropoulos B (1997) Rad51 immunocytology in rat and mouse spermatocytes and oocytes. Chromosoma 106:207-215

Murr R, Loizou JI, Yang YG, Cuenin C, Li H, Wang ZQ, Herceg Z (2006) Histone acetylation by Trrap-Tip60 modulates loading of repair proteins and repair of DNA double-strand breaks. Nat Cell Biol 8:91-99

Neale MJ, Keeney S (2006) Clarifying the mechanics of DNA strand exchange in meiotic recombination. Nature 442:153-158

Parra MT, Gomez R, Viera A, Llano E, Pendas AM, Rufas JS, Suja JA (2009) Sequential assembly of centromeric proteins in male mouse meiosis. PLoS Genet 5:e1000417

Peters AH, Plug AW, van Vugt MJ, de Boer P (1997) A drying-down technique for the spreading of mammalian meiocytes from the male and female germline. Chromosome Res 5:66-68

Pigozzi MI (2001) Distribution of MLH1 foci on the synaptonemal complexes of chicken oocytes. Cytogenet Cell Genet 95:129 133

Pigozzi MI, Solari AJ (1998) Germ cell restriction and regular transmission of an accessory chromosome that mimics a sex body in the zebra finch, Taeniopygia guttata. Chromosome Res 6:105-113

Pigozzi MI, Solari AJ (2005) The germ-line-restricted chromosome in the zebra finch: recombination in females and elimination in males. Chromosoma 114:403-409

Plug AW, Peters AH, Keegan KS, Hoekstra MF, de Boer P, Ashley T (1998) Changes in protein composition of meiotic nodules during mammalian meiosis. J Cell Sci 111(Pt 4):413-423

Roeder GS (1997) Meiotic chromosomes: it takes two to tango. Genes Dev 11:2600-2621

Rogakou EP, Pilch DR, Orr AH, Ivanova VS, Bonner WM (1998) DNA double-stranded breaks induce histone H2AX phosphorylation on serine 139. J Biol Chem 273:5858-5868

Rogakou EP, Nieves-Neira W, Boon C, Pommier Y, Bonner WM (2000) Initiation of DNA fragmentation during apoptosis induces phosphorylation of H2AX histone at serine 139. J Biol Chem 275:9390-9395

Scherthan H (2007) Telomere attachment and clustering during meiosis. Cell Mol Life Sci 64:117-124

Schoenmakers S, Wassenaar E, van Cappellen WA, Derijck AA, de Boer P, Laven JS, Grootegoed JA, Baarends WM (2008) Increased frequency of asynapsis and associated meiotic silencing of heterologous chromatin in the presence of irradiationinduced extra DNA double strand breaks. Dev Biol 317:270281

Schoenmakers S, Wassenaar E, Hoogerbrugge JW, Laven JS, Grootegoed JA, Baarends WM (2009) Female meiotic sex chromosome inactivation in chicken. PLoS Genet 5:e1000466 
Shahbazian MD, Grunstein M (2007) Functions of site-specific histone acetylation and deacetylation. Annu Rev Biochem $76: 75-100$

Sluss HK, Davis RJ (2006) H2AX is a target of the JNK signaling pathway that is required for apoptotic DNA fragmentation. Mol Cell 23:152-153

Solari AJ (1992) Equalization of $\mathrm{Z}$ and $\mathrm{W}$ axes in chicken and quail oocytes. Cytogenet Cell Genet 59:52-56

Terradas M, Martin M, Tusell L, Genesca A (2009) DNA lesions sequestered in micronuclei induce a local defective-damage response. DNA Repair 8:1225-1234

Tres LL (1977) Extensive pairing of the XY bivalent in mouse spermatocytes as visualized by whole-mount electron microscopy. J Cell Sci 25:1-15

Turner JM (2007) Meiotic sex chromosome inactivation. Development 134:1823-1831

Turner JM, Mahadevaiah SK, Ellis PJ, Mitchell MJ, Burgoyne PS (2006) Pachytene asynapsis drives meiotic sex chromosome inactivation and leads to substantial postmeiotic repression in spermatids. Dev Cell 10:521-529

Turner JM, Mahadevaiah SK, Fernandez-Capetillo O, Nussenzweig A, Xu X, Deng CX, Burgoyne PS (2005) Silencing of unsynapsed meiotic chromosomes in the mouse. Nat Genet 37:41-47
Vagnarelli P, Earnshaw WC (2004) Chromosomal passengers: the fourdimensional regulation of mitotic events. Chromosoma 113:211-222

Viera A, Calvente A, Page J, Parra MT, Gomez R, Suja JA, Rufas JS, Santos JL (2004a) X and B chromosomes display similar meiotic characteristics in male grasshoppers. Cytogenet Genome Res 106:302-308

Viera A, Santos JL, Page J, Parra MT, Calvente A, Cifuentes M, Gomez R, Lira R, Suja JA, Rufas JS (2004b) DNA double-strand breaks, recombination and synapsis: the timing of meiosis differs in grasshoppers and flies. EMBO Rep 5:385-391

Vigodner M (2009) Sumoylation precedes accumulation of phosphorylated $\mathrm{H} 2 \mathrm{AX}$ on sex chromosomes during their meiotic inactivation. Chromosome Res 17:37-45

Vigodner M, Morris PL (2005) Testicular expression of small ubiquitin-related modifier-1 (SUMO-1) supports multiple roles in spermatogenesis: silencing of sex chromosomes in spermatocytes, spermatid microtubule nucleation, and nuclear reshaping. Dev Biol 282:480-492

Zhou W, Wang X, Rosenfeld MG (2009) Histone H2A ubiquitination in transcriptional regulation and DNA damage repair. Int $\mathrm{J}$ Biochem Cell Biol 41:12-15

Zickler D (2006) From early homologue recognition to synaptonemal complex formation. Chromosoma 115:158-174 